\title{
Detection of three-dimensional surfaces from optic flow: The effects of noise
}

\author{
GEORGE J. ANDERSEN and A. PAIGE WUESTEFELD \\ University of California, Riverside, California
}

\begin{abstract}
Previous research (Andersen, 1989) has suggested that the recovery of 3-D shape from nonsmooth optic flow (motion transparency) can be performed by segregating surfaces according to the distributions of velocities present in the flow field. Five experiments were conducted to examine this hypothesis in a surface detection paradigm and to determine the limitations of human observers to detect 3-D surfaces in the presence of noise. Two display types were examined: a flow field that simulated a surface corrugated in depth and a flow field that simulated a random volume. In addition, two types of noise were examined: a distribution of noise velocities that overlapped or did not overlap the velocity distribution that defined the surface. Corrugation frequency and surface density were also examined. Detection performance increased with decreasing corrugation frequency, decreasing noise density, and decreasing surface density. Overall, the subjects demonstrated remarkable tolerance to the presence of noise and, for some conditions, could discriminate surface from random conditions when noise density was twice the surface density. Discrimination accuracy was greater for the nonoverlapping than for the overlapping noise, providing support for an analysis based on the distribution of velocities.
\end{abstract}

Optic flow, the perspective transformation of visible feature points during motion of the observer or objects in the environment, can be a useful source of information for the the recovery of 3-D shape and the layout of a scene (Gibson, 1950, 1966; Helmholtz, 1867/1962; von Kries, 1910/1962). Considerable research has examined the ability of human observers to recover the shape and depth of objects in a scene from optic flow. Studies have demonstrated the effectiveness of optic flow in providing the curvature and shape of a surface (Cornilleau-Pérès \& Droulez, 1989; Eby, 1992; Norman \& Lappin, 1992; Rogers \& Graham, 1979; Todd, 1984), the orientation in depth of a surface (Braunstein \& Andersen, 1981), the slant of a surface (Braunstein \& Payne, 1969), and the depth order and relative depth between surfaces (Andersen, 1989).

An important assumption in this research is that sufficient information is present in the display for the perception of a surface. Although considerable research has examined the minimal conditions for the perception of a surface from binocular disparity (e.g., Uttal, 1975, 1983, 1985, 1987, 1988; Uttal, Davis, Welke, \& Kakarala, 1988), there has been relatively little research examining the minimal conditions for surface detection from optic flow. In a recent study examining the minimal conditions

This research was supported by National Science Foundation Grant BNS 9021081 . The authors would like to thank Myron L. Braunstein, Jan Koenderink, and Jim Todd for comments on an earlier draft. We would also like to thank Myron L. Braunstein for beneficial discussions regarding the design of Experiment 5. Reprint requests should be sent to G. J. Andersen, Department of Psychology, University of California, Riverside, California 92521. for the detection of 3-D surfaces from optic flow (Andersen, 1991, 1993), subjects were presented with displays simulating points undergoing rigid horizontal translation. In one condition, the points were positioned on a surface corrugated in depth (see Figure 1). In a second condition, the velocities of points used to define the surface were randomly repositioned in the image. The displays in both of these conditions had identical velocities; however, in one case the velocities defined a 3-D surface, whereas in the other case the velocities defined a random volume of points. On each trial, the subjects were presented with either a surface display or a random-volume display and were asked to indicate whether or not the points defined a 3-D surface. The texture density of the display and the corrugation frequency were varied. Detection performance was above chance, even at relatively low density levels $\left(0.41 \mathrm{dots} / \mathrm{deg}^{2}\right)$. Detection accuracy decreased with an increase in corrugation frequency and a decrease in the corrugation amplitude of the surface.

The purpose of the present research was to examine the ability of human observers to detect the presence of a surface within noise (velocities unrelated to the surface). Surface detection in the presence of noise can be considered to be a case of motion transparency. Recent research has demonstrated that human observers are able to detect multiple transparent overlapping frontoparallel surfaces from optic flow (Andersen, 1989). The subjects were presented with displays in which the number of overlapping velocity fields in the image, specifying different transparent surfaces separated in depth, varied between one and five. The subjects, when asked to indicate the number of transparent planes, were able to identify up to three overlapping surfaces for both horizontal and depth translations. 


\section{Corrugated Surface}
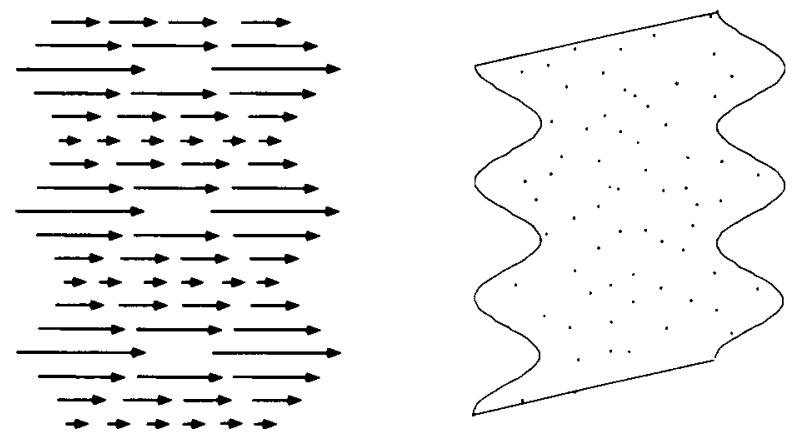

Random Volume
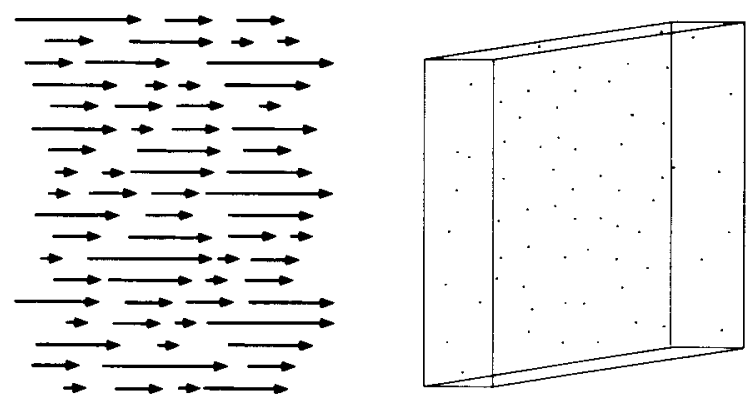

Figure 1. Examples of two optic flow fields and the simulated 3-D configurations. The top flow field is the velocity field produced by perspective horizontal translation of a 3-D surface corrugated in depth according to a sine function. The bottom flow field is a randomized version of the top flow field, with the velocities repositioned randomly in the image. The velocity vectors are regularly spaced along the vertical axis for purposes of illustration. In the present experiments, the velocities were randomly positioned in the image.

The ability of observers to detect multiple overlapping velocity fields demonstrates that human observers do not require smoothness of the velocity field for the perception of 3-D surfaces. Other studies (De Bruyn, 1992; Gerbino \& Bernetti, 1984; van Doorn \& Koenderink, 1982a) have examined this issue in the context of 2-D motion detection. For example, van Doorn and Koenderink (1982a, $1982 b$ ) found that observers could detect two overlapping velocity fields presented within a scintillating noise pattern. Studies have also demonstrated the ability of observers to segregate two overlapping velocity fields translating in different directions (Adelson \& Movshon, 1982; Snowden, 1990). These studies demonstrate that human observers can segregate overlapping velocity signals based on differences in velocity and direction of motion.

Longuet-Higgins and Prazdny (1980) proposed an analysis capable of segregating velocities that define a surface from velocities that do not define the surface. According to their model, the depth separation between two points aligned along the same visual direction can be determined from the difference in magnitude of the velocities. However, this analysis will only be useful for velocities of feature points that momentarily occupy the same or nearby coordinates in the image. The probability of this occurrence was quite low across conditions in which human observers could recover 3-D structure from nonsmooth optic flow (Andersen, 1989), suggesting that this analysis is not sufficient to account for human performance in these conditions.

One method that the visual system might use to segregate velocities that define a specific 3-D surface from other velocities in the flow field is to analyze the distribution of velocities present in the display (Andersen, 1989). This is possible because surfaces that are separated in depth will be specified by separate distributions of velocities as a result of motion parallax. Once the separate distributions of velocities are extracted, other analyses concerned with 3-D surface recovery, such as the divergence, curl, and deformation analyses proposed by Koenderink (1986) and Koenderink and van Doorn (1976), could be applied to the separate distributions.

Consider the rigid horizontal translation of a transparent corrugated surface embedded within a volume of random points (see Figure 2A). Because the surface and noise points occupy the same region along the depth axis, the distribution of velocities for surface and noise points will overlap (see Figure 3). We will refer to this stimulus configuration as overlapping noise. In addition, consider the rigid horizontal translation of a transparent corrugated surface and two transparent frontoparallel surfaces (noise) separated in depth from the corrugated surface (see Figure 2B). Because the corrugated surface and frontoparallel surfaces occupy different regions along the depth axis, the distribution of velocities for surface and noise points will not overlap, as shown in Figure 3. We will refer to
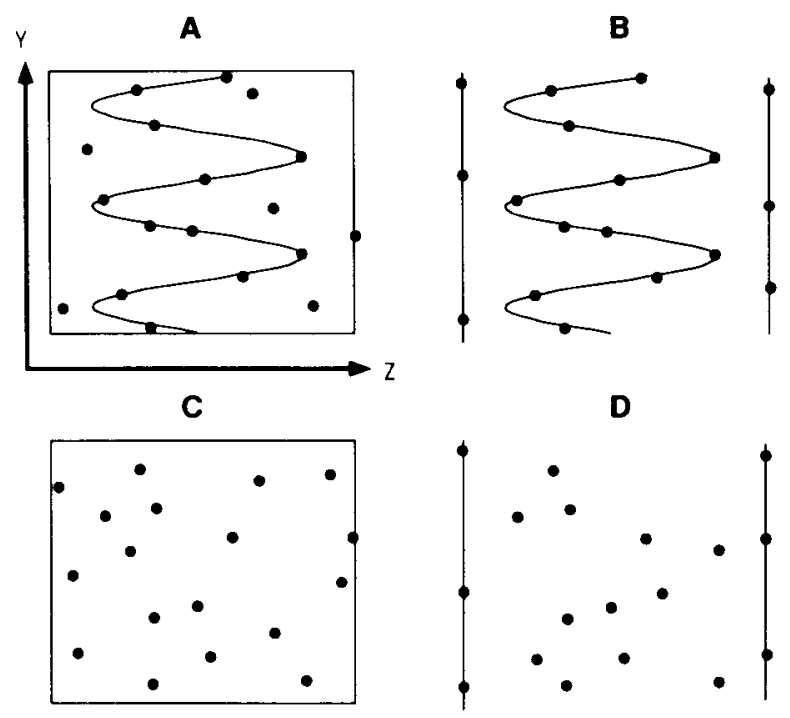

Figure 2. Side views illustrating the 3-D configurations and types of noise examined in the present study. Panels $A$ and $B$ depict corrugated surface displays and $C$ and $D$ depict random volume displays. Panels $A$ and $C$ depict overlapping noise (examined in Experiments 1 and 2) and $B$ and $D$ depict nonoverlapping noise (examined in Experiments 3 and 4). 

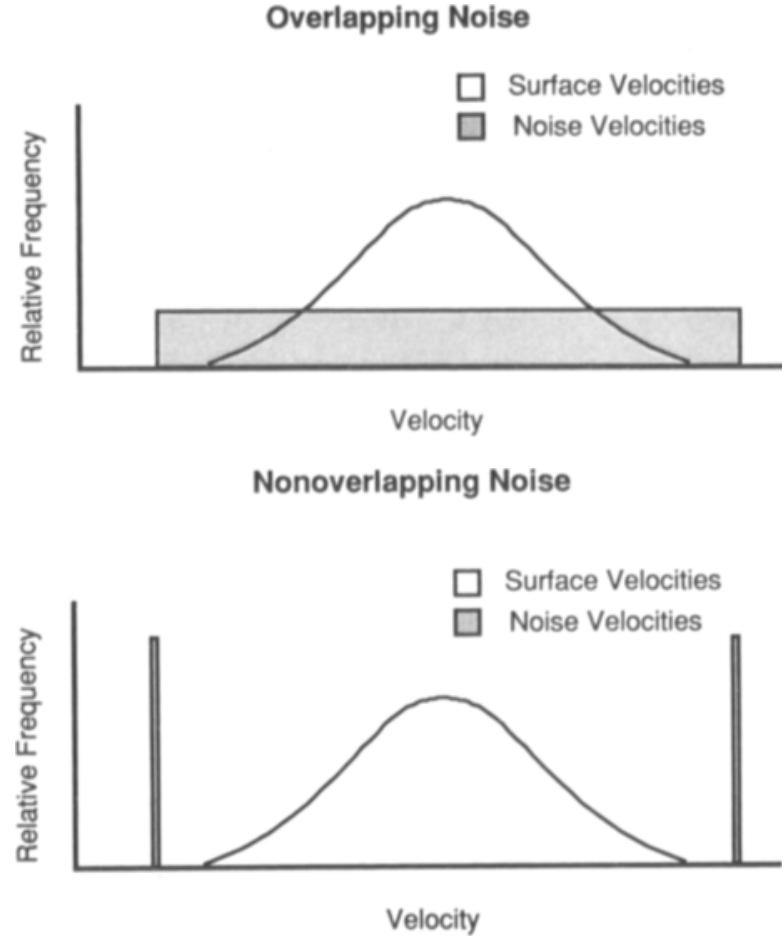

Figure 3. The two types of noise (overlapping and nonoverlapping) examined in the present experiments, illustrated in terms of theoretical distributions of velocities present in the display. Overlapping noise was examined in Experiments 1 and 2, whereas nonoverlapping noise was examined in Experiments 3 and 4.

this second stimulus configuration as nonoverlapping noise. This condition is comparable to the display simulating three surfaces examined by Andersen (1989).

The noise velocities for the overlapping and nonoverlapping conditions have the same range and mean. The primary difference between these conditions is whether the distributions of noise and surface velocities overlap. If subjects use an analysis based on the separation between distributions of velocities, then we predict greater accuracy in detecting the presence of a 3-D surface when nonoverlapping noise is present in the display than when overlapping noise is present.

The purpose of the present research was to examine the ability of human observers to detect the presence of a surface from optic flow when noise was present and to determine whether the analysis of nonsmooth optic flow is based on the distribution of velocities present in the display. Subjects were presented with displays simulating horizontal translation of points positioned randomly in the image. On some trials, the points were positioned on a simulated corrugated surface that varied in depth according to a sine function. Horizontal translation of the surface resulted in an optic flow field that varied in velocity according to the sine function (see Figure 1). On other trials, the velocities were randomly repositioned vertically in the image (see Figure 1). This resulted in a flow field that simulated points randomly positioned in a volume. For each display, the subjects were asked to indicate whether or not the pattern of moving points formed a 3-D curved surface. In addition to these display conditions, we examined the two types of noise discussed above. In Experiments 1 and 2, we examined surface detection in the presence of overlapping noise. Both corrugation frequency and surface density have been found (Andersen, 1991, 1993; Uttal, 1983, 1985) to be critical factors for surface detection under minimal conditions. In Experiment 1 , we examined the effects of variations in the corrugation frequency and the density of noise points. The strength of the noise in any given display, relative to the strength of the signal (velocities specifying the surface), can be expressed as the ratio of noise density to surface density. An important issue is whether a constant value of this ratio implies a constant level of detection of surfaces in the presence of noise. In order to examine this issue, we varied surface density and noise density in Experiment 2, for a limited set of corrugation frequencies. In Experiments 3 and 4, we examined surface detection in the presence of nonoverlapping noise for the surface density, noise density, and corrugation frequency conditions examined in Experiments 1 and 2, respectively. In Experiment 5, we directly compared surface detection from optic flow in the presence of either overlapping or nonoverlapping noise for a subset of the surface density, noise density, and corrugation frequency conditions examined in Experiments 1-4.

\section{EXPERIMENT 1}

The purpose of Experiment 1 was to examine the ability of human observers to detect the presence of a surface in overlapping noise. In order to assess the effects of noise on surface detection, we systematically varied the texture density of the noise points while maintaining a constant texture density for the surface. In addition, the effect of surface structure was examined by varying the spatial frequency of the corrugated pattern. Increasing the number of cycles of the simulated corrugated surface resulted in a change in both total simulated surface area and local 3-D curvature of the surface. Previous research by Andersen $(1991,1993)$ showed that variations in the frequency of the simulated corrugated surface is important for 3-D surface detection.

\section{Method}

Subjects. The subjects were 4 graduate students and 1 undergraduate student at the University of California, Riverside who were paid for their participation. All the subjects were familiar with optic flow displays but were naive concerning the purpose of the experiment. They all had normal or corrected-to-normal vision.

Design. Three independent variables were examined: type of display (corrugated surface or random volume), the density of the noise points $\left(0,1.04,2.4,4.8,9.6\right.$, and $\left.19.2 \mathrm{dots} / \mathrm{deg}^{2}\right)$, and spatial frequency of the corrugated surface $(0.52,0.79$, and 1.05 cycles per degree [cpd] of visual angle).

Stimuli. The stimuli were computer-generated displays that simulated points positioned either on a 3-D surface or randomly within 
a volume (see Figure 1). The surface displays were generated in the following manner. Points were randomly positioned in the image and translated along the horizontal axis; velocities were assigned to the points according to a sine function. Specifically, the position change of a point in the image per frame $(i)$ was determined by using the following equation:

$$
i=a \sin \left(f 2 \pi y / y_{\max }\right)+a+b,
$$

where $a$ is the amplitude of the corrugated surface, $b$ is the minimum horizontal motion per frame, $f$ is the frequency of the corrugated surface, $y$ is the vertical coordinate (in pixel units) of the point, and $y_{\max }$ is the maximum $y$ value. This simulated a sinusoidal surface in depth that was corrugated along the vertical axis. To produce the random volume conditions, the points and velocities of the corrugated surface displays were randomly reassigned to different vertical positions in the image (see Figure 1). This resulted in a velocity field in which the velocities varied randomly along the vertical axis. These two display conditions had identical distributions of velocities in the image; however, in the noise condition, the velocities did not vary with the vertical position. Velocities for both the surface and random conditions ranged between $1.95 \% \mathrm{sec}$ and $2.84 \% \mathrm{sec}$. The density of both the surface and random displays was $9.6 \mathrm{dots} / \mathrm{deg}^{2}$.

The effect of noise velocities on the detection of 3-D surfaces was examined in the following manner. Noise points were randomly distributed within the image, and were randomly assigned a velocity ranging between $0.76 \% \mathrm{sec}$ and $4.04 \% \mathrm{sec}$. Each noise point maintained the same velocity for the duration of the display. The number of noise points added was determined by the noise density level (see Design). Noise points were added to both surface and random displays.

The displays subtended an $8.74^{\circ}$ square region. The size of the dots was $0.51^{\prime}$ of arc. Whenever a dot was projected outside the image area during the horizontal translation, it was relocated to the opposite horizontal position in the image at the same $y$ coordinate. The display was updated at $27 \mathrm{~Hz}$. The displays oscillated once (motion left to right and right to left) over a 96 -frame cycle. The display duration was $3.55 \mathrm{sec}$.

Apparatus. The displays were generated on an Evans and Sutherland ESV30 graphics workstation (resolution 1,280×1,024). An eye patch was used for monocular viewing.

Procedure. The subjects participated individually. They were informed that they would be viewing a series of displays of moving white dots. On some trials, the dots would appear to form a corrugated 3-D surface embedded within noise (points that did not belong to the surface). On other trials, the dots would appear as a random 3-D pattern. The subjects' task was to determine whether a subset or all of the points in the display defined a 3-D curved surface. The subjects used the keypad on the keyboard to respond. They were asked to respond at the end of each display by pressing " 1 " on the keyboard if they perceived a 3-D surface or " 0 " if they did not perceive a 3-D curved surface.

The subjects viewed the displays monocularly in a dark room at a distance of $1.7 \mathrm{~m}$. They participated in three 60 -min sessions, run on separate days. Each session consisted of 10 blocks; each block consisted of a single presentation of each of the 36 displays in random order. Thus, each subject viewed 30 trials of each display condition for a total of 1,080 trials. An additional block of trials was run at the beginning of the first session and was used as practice. No feedback was provided to the subjects during either the practice or test trials. At the end of the experiment, they were asked whether they had used any strategy to respond to the displays.

\section{Results and Discussion}

All the subjects reported a compelling perception of depth from the displays, with some of the displays pro- ducing a distinct impression of a 3-D surface. The dependent measure was the proportion of correct responses. The results are presented in Figure 4. Each data point is the average of 30 responses from 5 subjects. In order to assess the effects of noise on surface detection, we plotted the results in terms of the ratio of the noise to signal (surface) density. Thus, a noise/signal density ratio of 0 indicates that no noise points were present in the display, whereas a ratio of 2 indicates that the density of noise points was twice the density used to define the surface. As is shown in Figure 4, the subjects were consistently correct at responding to the random display condition. The mean proportion correct was greater than .94 , regardless of variations in the noise/signal ratio of the display. These
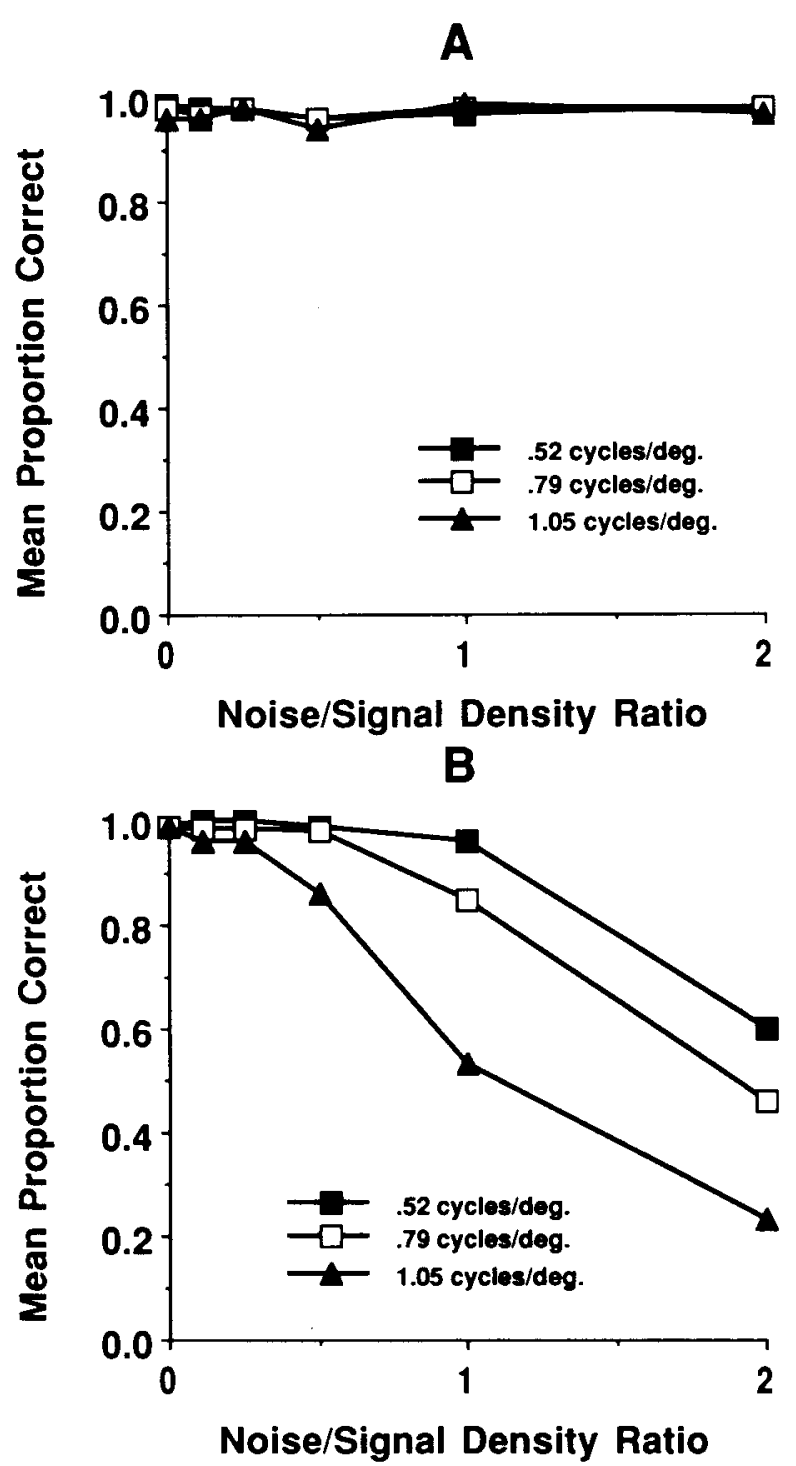

Figure 4. Mean proportion correct as a function of noise/signal density ratio for the random $(A)$ and surface $(B)$ conditions. Separate curves are plotted for surfaces with corrugation frequencies of $.52, .79$, and 1.05 cpd. 
results indicate that the subjects were not merely guessing on random-display trials. Had the subjects adopted this strategy, performance would have been at chance $(50 \%)$; instead, these results demonstrate a response bias for random displays. When subjects are presented with a random display, they will almost always report that no surface is present.

A different pattern of results was obtained for the surface display trials (see Figure 4). For these trials, mean proportions correct decreased with an increase in the noise/signal ratio of the display. The mean proportions correct for the $0,0.10,0.25,0.5,1.0$, and 2.0 noise/signal ratios were $.99, .982, .982, .943, .78$, and .43 , respectively. These results demonstrate a remarkable tolerance for the presence of noise in these displays. The subjects' performance was below chance for only the 2.0 noise/ signal ratio condition. This condition represents a display in which twice as many noise points, as compared with surface points, were present.

Detection performance also decreased with an increase in the spatial frequency of the corrugated surface. The mean proportions correct for the $0.52-, 0.79$, and 1.05 cpd corrugated surfaces were $.923, .875$, and .755 . This result is similar to the finding by Andersen (1991, 1992), who found a decrease in surface detection as a function of the spatial frequency of the corrugated surface when no noise points were present in the display. The present results extend this finding to conditions in which noise points were present.

There was also a performance tradeoff between the spatial frequency of the corrugated surface and the noise/ signal density ratio. As is shown in Figure 4, detection performance decreased at a greater rate, as a function of an increase in the noise/signal density ratio, for higher corrugation frequencies than for lower corrugation frequencies. These results demonstrate a greater tolerance for the presence of noise for low-spatial-frequency corrugated surfaces. Indeed, accuracy at the 2.0 noise/signal density ratio was .62 for the 0.52 -cpd surface. However, for the 1.05-cpd corrugated surface, performance for the 2.0 noise/signal density level was .22. A decrease in the noise/signal ratio resulted in an increase in detection performance, with near-perfect performance obtained for the .10 noise/signal density ratio across all spatial frequency surfaces examined.

When asked during debriefing about the use of response strategies, all the subjects reported that their responses were based on the 3-D appearance of the displays.

\section{EXPERIMENT 2}

In Experiment 1, accuracy in detecting a 3-D corrugated surface was determined by the frequency of the corrugation and density of the noise points. The purpose of Experiment 2 was to determine the effects of these variables when the texture density of the surface was varied. Previous research by Andersen $(1991,1992)$ showed that a decrease in the density of points defining a surface in the absence of noise resulted in a decrease in detection performance. In Experiment 2, we examined the effects of overlapping noise when the texture density that specified a 3-D surface was reduced.

\section{Method}

Subjects. The 5 subjects who participated in Experiment $I$ also participated in Experiment 2.

Design. Four independent variables were examined: type of display (corrugated surface or random volume), the density of the surface points $\left(2.4,4.8\right.$, and $\left.9.6 \mathrm{dots} / \mathrm{deg}^{2}\right)$, the density of the noise points $\left(0,2.4,4.8,9.6\right.$, and $\left.19.2 \mathrm{dots} / \mathrm{deg}^{2}\right)$, and the spatial frequency of the corrugated surface $(0.52$ or $1.05 \mathrm{cpd})$.

Stimuli. The stimuli were similar to the displays used in Experiment 1 . The displays simulated a 3-D corrugated surface or a random 3-D volume specified by optic flow. The velocities used for the surface and random conditions and for the noise were identical to those used in Experiment 1.

Apparatus and Procedure. The apparatus and procedure that were used in Experiment 1 were used.

\section{Results and Discussion}

The results are presented in Figure 5. Each data point is the average of 30 responses from 5 subjects. The results are plotted in terms of the noise/signal density ratio. As is shown in Figure 5, the subjects were consistently correct in detecting the random displays. This result replicates the high proportion of correct responses found for the random display conditions in Experiment 1.

The results for the surface displays are shown in Figures $5 \mathrm{C}$ and $5 \mathrm{D}$. For the surface display conditions, overall performance was worse for the high-frequency $(1.05 \mathrm{cpd})$ surface displays than for the low-frequency $(0.52 \mathrm{cpd})$ surface displays. The mean proportions correct for the 1.05 - and 0.52 -cpd surfaces were .39 and .66 , respectively, which replicates the effect of frequency obtained in Experiment 1. This result occurred across a wide range of noise/signal density values and varied as a function of surface density. For example, consider the results for the noise/signal ratio of 1 for the 0.52 -cpd frequency display. The mean proportions correct for the 2.4-, 4.8-, and 9.6dots/deg ${ }^{2}$ surface densities were $.26, .44$, and .80 , respectively. A similar pattern of results occurred for the 1.05cpd frequency display. For the noise/signal density ratio of 1 , the mean proportions correct for the 2.4-, 4.8-, and 9.6-dots/ $/ \mathrm{deg}^{2}$ surface densities were $.07, .12$, and .26 . In addition, the results for these combined conditions of surface density and frequency indicate that detection performance varied according to the surface density.

The present results indicate several important findings. First, the detection of 3-D surfaces from optic flow, when noise is present, varies with the noise/signal density ratio. This pattern of results was found for both corrugation frequencies examined. Overall, performance decreased with an increase in this ratio, demonstrating that the detection of a surface when presented in noise can $\alpha c-$ cur within a limited range of noise.

However, the effect of the noise/signal density ratio on detection accuracy is not solely determined by variations in the texture density of the noise. A second finding was 

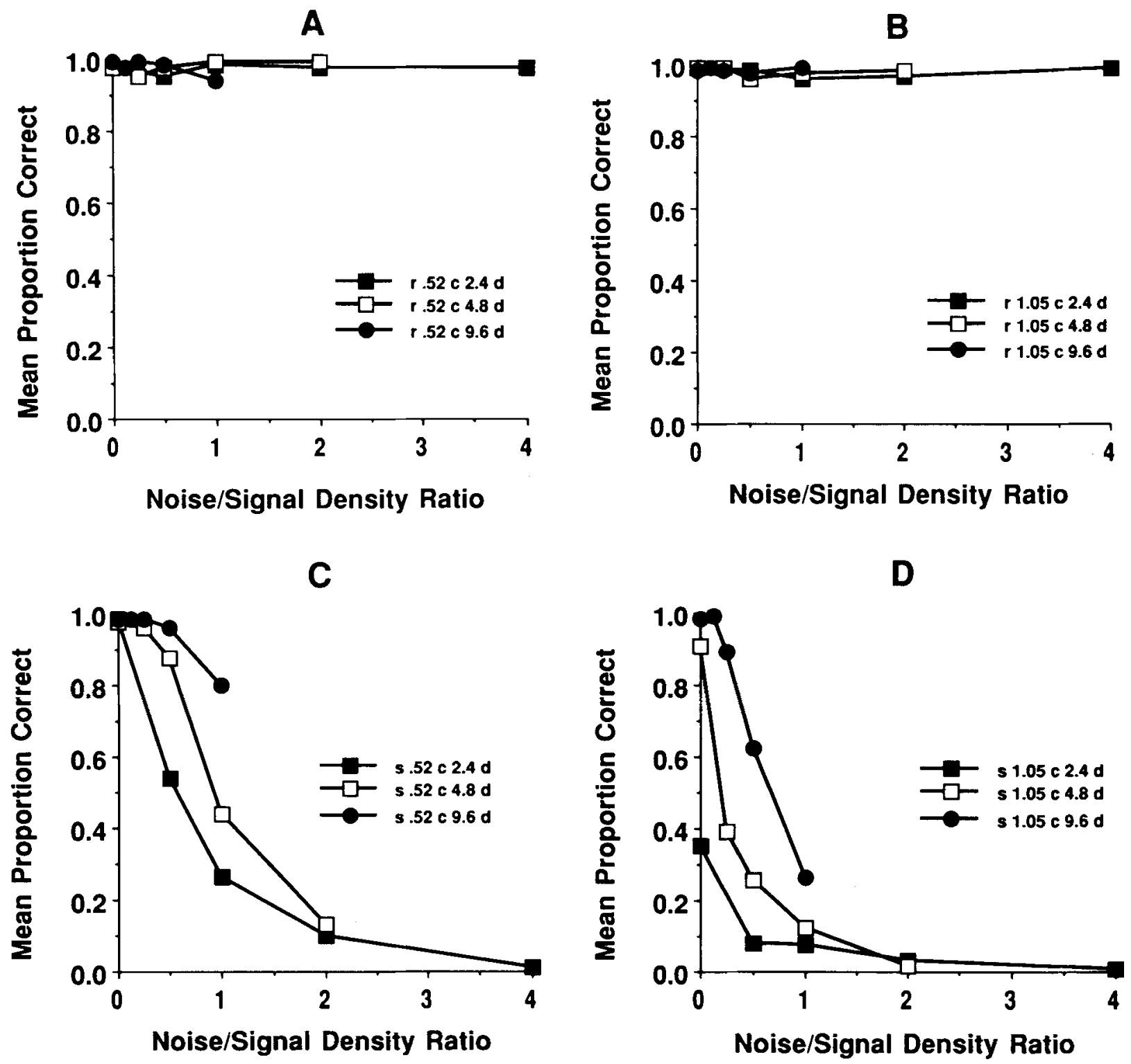

Figure 5. Mean proportion correct as a function of noise/signal density ratio for random $(A$ and $B)$ and surface $(C$ and $D)$ conditions. Separate curves are plotted for different random (r), surface (s), corrugations (cycles per degree; c), and surface density (dots per degree ${ }^{2}$; d).

that the effect of noise varied as a function of the texture density of the surface. The subjects demonstrated greater accuracy for surfaces with a higher texture density. This finding was not the result of insufficient density to detect the surface when noise was not present. Examination of Figure 5C for the 0 noise/signal density level indicates that the subjects had perfect performance in detecting the 0.52 -cpd surface when noise was not present.

Third, corrugated surfaces with a lower frequency can be detected when more noise is present than corrugated surfaces with a higher frequency. Surfaces with greater corrugation frequency also have a greater rate of curvature change. Previous research (Andersen, 1993) has suggested that detection of surfaces from optic flow is based on estimates of curvature change. This suggests that detection performance of surfaces embedded in noise may be more difficult for surfaces with greater rates of curvature change than for surfaces with lower rates of curvature change.

\section{EXPERIMENT 3}

In Experiments 1 and 2, we investigated the detection of 3-D surfaces embedded within a random volume of noise points. For this condition, the distribution of noise velocities overlapped the distribution of velocities that specified the surface. As discussed earlier, noise can also be added by introducing points with velocities that do not 
overlap with the distribution of velocities that define the surface. Noise from nonoverlapping velocity distributions results in displays that are comparable to some of the display conditions examined by Andersen (1989).

The subjects were presented with displays simulating corrugated sinusoidal surfaces similar to those examined in Experiments 1 and 2. In Experiment 3, the noise points translated horizontally at velocities that were either greater or less than the velocities that defined the corrugated surface. This simulation was consistent with the corrugated surface positioned, along the depth axis, between two noise surfaces (see Figure 3). In addition, this resulted in a display in which the distribution of velocities of noise points did not overlap the distribution of velocities of surface points. If the subjects were to employ an analysis based on the distribution of velocities - in which the separation of distributions of velocities of noise and surface points is important-then we would expect greater accuracy for nonoverlapping noise than for overlapping noise (examined in Experiments 1 and 2). In order to compare results for overlapping and nonoverlapping noise, the frequency and noise densities that were examined in Experiment 1 were also examined in Experiment 3.

\section{Method}

Subjects. The subjects were the 5 subjects who had participated in Experiments 1 and 2. They were paid for their participation.

Design. Three independent variables were examined: type of display (comugated surface or random volume), the density of the noise points $\left(0,1.04,2.4,4.8,9.6\right.$, and $\left.19.2 \mathrm{dots} / \mathrm{deg}^{2}\right)$, and the spatial frequency of the corrugated surface $(0.52,0.79$, and $1.05 \mathrm{cpd}$ of visual angle)

Stimuli. The stimuli were similar to the displays used in Experiments 1 and 2, except for the type of noise that was added. The displays simulated a 3-D corrugated surface or a random 3-D volume specified by optic flow. The surface and random velocities were generated in the same manner as in Experiments 1 and 2. Noise points were randomly positioned within the image and were randomly assigned with equal probability to a velocity of $0.76 \% \mathrm{sec}$ or $4.04 \% \mathrm{sec}$. These velocities were the maximum and minimum noise velocities examined in Experiments 1 and 2. Thus, the mean velocity and range of velocities were identical to the stimuli used in Experiments 1 and 2.

Apparatus and Procedure. The apparatus and procedure were the same as those used in Experiment 1.

\section{Results and Discussion}

The results are presented in Figure 6. Each data point is the average of 30 responses from 5 subjects. As is shown in Figure 6A, the subjects were consistently correct in detecting the random displays. The mean proportion correct was greater than .94 , regardless of variations in the noise/signal ratio of the display, and are similar to the results obtained for random displays in Experiments 1 and 2.

For surface display trials (shown in Figure 6B), the mean proportion correct decreased with an increase in the noise/signal ratio of the display. As is shown in Figure 6B, detection performance decreased with an increase in the spatial frequency of the corrugated surface. The mean proportions correct for the $0.52-, 0.79-$, and 1.05 -cpd cor-
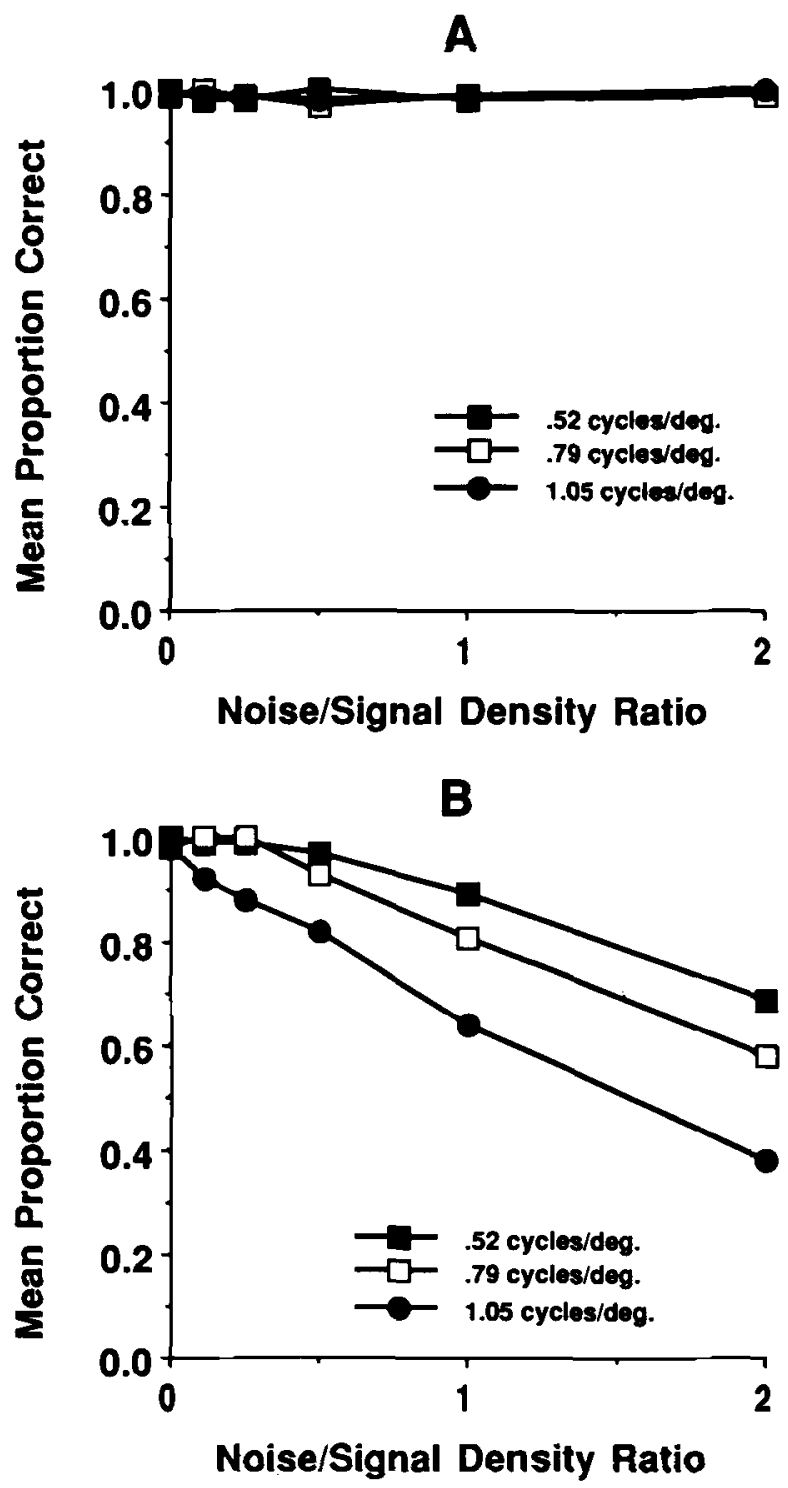

Figure 6. Mean proportion correct as a function of noise/signal density ratio for the random (A) and surface (B) conditions. Separate curves are plotted for surfaces with corrugation frequencies of $.52, .79$, and 1.05 cpd.

rugations were $.92, .88$, and .77 , respectively. These results are similar to the results obtained in Experiment 1 $(.923, .875$, and .755$)$, and demonstrate that an increase in the spatial frequency of the corrugated surface resulted in a decrease in detection performance.

Performance was also affected by the noise/signal density ratio. The mean proportions correct for the $0, .1, .25$, $.5,1$, and 2 noise/signal density ratios were $.98, .97, .95$, $.91, .78$, and .55 , respectively. The results from Experiment 1 for these levels were $.99, .98, .98, .94, .78$, and .43 , demonstrating that an increase in the noise/signal ratio resulted in a decrease in detection performance. However, the results did not demonstrate a noticeable difference in performance between Experiments 1 and 3. 
Detection performance decreased more rapidly with increasing noise/signal density ratio for higher corrugation frequencies than for lower corrugation frequencies (see Figure 6B). These results demonstrate a greater tolerance for the presence of noise for lower spatial frequency corrugations.

An important goal of Experiment 3 was to compare detection performance for nonoverlapping and overlapping noise. The results discussed above indicate that little difference was found between the results obtained in Experiments 1 and 3 for frequency and noise/signal ratio. One reason for the similar levels of performance may be that the conditions were not sufficiently difficult to produce different results. In Experiment 2, detection accuracy was examined with a greater range of noise/signal density ratios. In Experiment 4, we examined surface detection, in the presence of nonoverlapping noise, across the range of noise/signal density ratios examined in Experiment 2.

\section{EXPERIMENT 4}

In Experiment 3, we examined the effects of nonoverlapping noise on the detection of 3-D surfaces. The pattern of results for nonoverlapping noise was similar to the results obtained for overlapping noise examined in Experiment 1 . The purpose of Experiment 4 was to examine the effects of nonoverlapping noise over a larger range of noise/signal density ratios by varying the surface density. In the present experiment, the levels of corrugation frequency, noise density, and surface density were identical to the levels examined in Experiment 2, in which overlapping noise was employed.

\section{Method}

Subjects. The subjects were the 5 subjects who had participated in Experiments 1, 2, and 3. They were paid for their participation.

Design. Four independent variables were examined: type of display (corrugated surface or random volume), the density of the surface points $\left(2.4,4.8\right.$, and $\left.9.6 \mathrm{dots} / \mathrm{deg}^{2}\right)$, the density of the noise points $\left(0,2.4,4.8,9.6\right.$, and $\left.19.2 \mathrm{dots} / \mathrm{deg}^{2}\right)$, and the spatial frequency of the corrugated surface $(0.52$ or $1.05 \mathrm{cpd})$.

Stimuli, Apparatus, and Procedure. The stimuli, apparatus, and procedure were the same as in Experiment 3.

\section{Results and Discussion}

The results are presented in Figure 7. Each data point is the average of 30 responses from 5 subjects. As is shown in Figures 7A and 7B, the subjects were consistently correct in detecting the random displays, which replicates the high proportion of correct responses found for the random-display conditions in Experiments 1, 2, and 3.

The results for the surface displays are presented in Figures 7C and 7D. Overall performance was worse for the high frequency than for the low frequency. The mean proportions correct for the 0.52 - and 1.05 -cpd corrugations were .842 and .55 , respectively. This result replicates the effects of corrugation frequency found in the previous three experiments. The effect of the noise/signal ratio on detection performance varied as a function of the density of surface points. Specifically, greater detection accuracy occurred for higher surface densities, compared with lower surface densities, across variations in the noise/ signal ratio. This pattern of results is similar to the results obtained in Experiment 2, in which overlapping noise was used.

A comparison of the results obtained from Experiments 2 and 4 is presented in Figure 8. This figure contrasts the results for overlapping and nonoverlapping noise obtained in Experiments 2 and 4, as a function of different combinations of surface frequency and density. As is shown in Figure 8, detection performance was greater for nonoverlapping than for overlapping noise across every comparison based on combinations of surface frequency and surface density. A comparison of overlapping and nonoverlapping noise, in terms of noise density, is shown in Figure 9. These results demonstrate decreased detection performance for overlapping noise, compared with nonoverlapping noise, as noise density increased. These results provide support for the hypothesis that the detection of 3-D surfaces in noise may be based on segregating surface and noise points on the basis of their velocity distributions. In Experiment 4 , we examined a larger range of noise/signal density conditions than we did in Experiments 1 and 3 . Thus, the lack of a difference for overlapping and nonoverlapping noise in those experiments may have been a result of the limited set of conditions examined.

\section{EXPERIMENT 5}

The results from Experiments 2 and 4 suggest that greater accuracy occurred for nonoverlapping than for overlapping noise. However, one problem with the noise conditions examined in these experiments was that, for overlapping noise, the points were randomly positioned within a volume, whereas for nonoverlapping noise, the points were randomly positioned on one of two frontoparallel surfaces. Thus, a potential problem with comparisons across these experiments is that the presence or absence of overlapping noise velocities was confounded with whether or not the noise velocities defined a structure. The presence of noise that defined a structure may have been beneficial, as interpolation algorithms (e.g., Grimson, 1981) could be used to aid in segregating surface velocities from noise velocities.

We conducted an additional experiment to address this issue. Two primary variables were examined: type of noise (overlapping or nonoverlapping) and noise configuration (random volume or two frontoparallel surfaces). Combinations of these two variables resulted in four noise configurations: overlapping volume noise, overlapping surface noise, nonoverlapping volume noise, and nonoverlapping surface noise. The velocity distributions of these four conditions are depicted in Figure 10. If the segregation of velocities is based on the overlap of velocity distributions of surface and noise points, then we would expect greater detection accuracy for nonoverlapping noise than for overlapping noise. However, if interpolation of a surface from noise velocities is important, then we would 
A
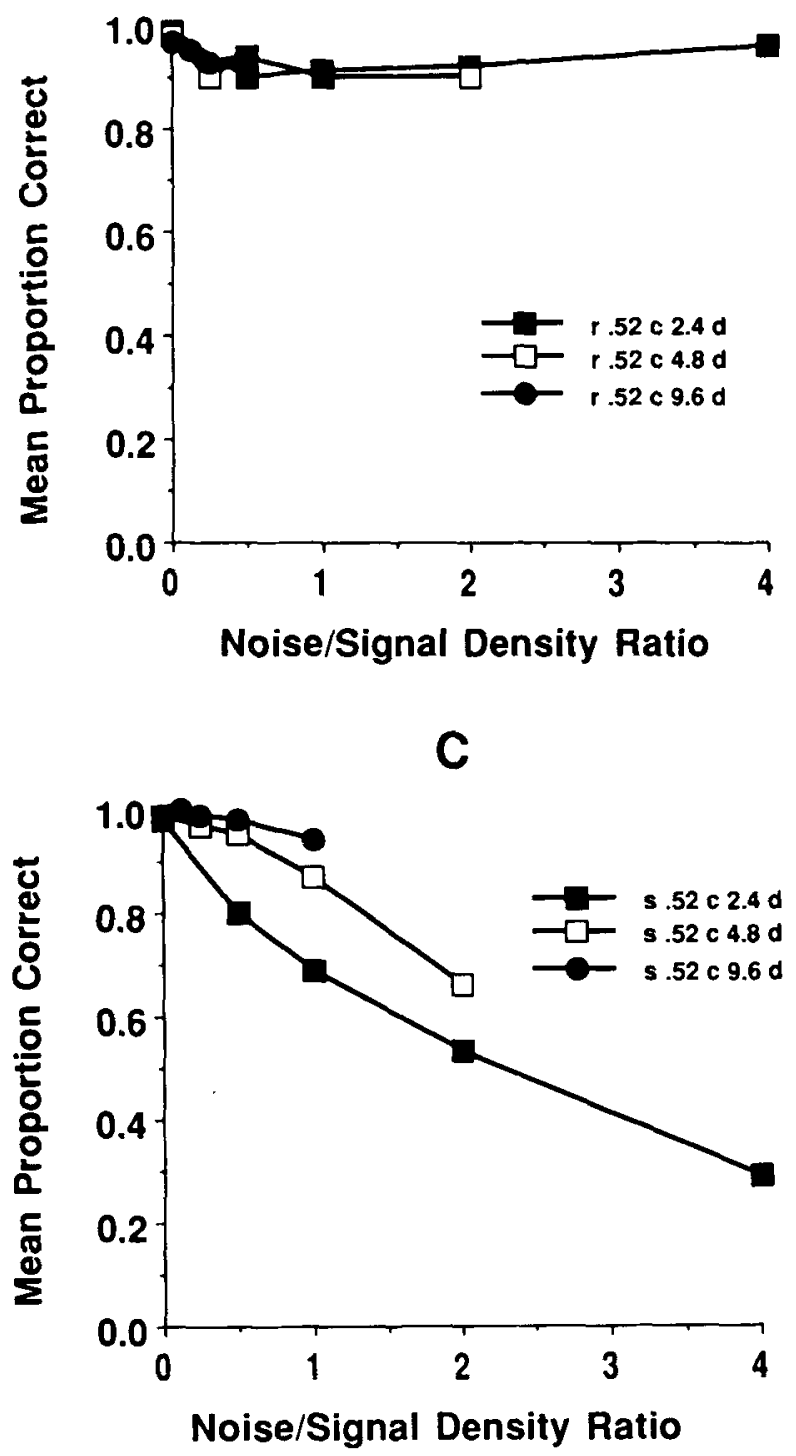

B
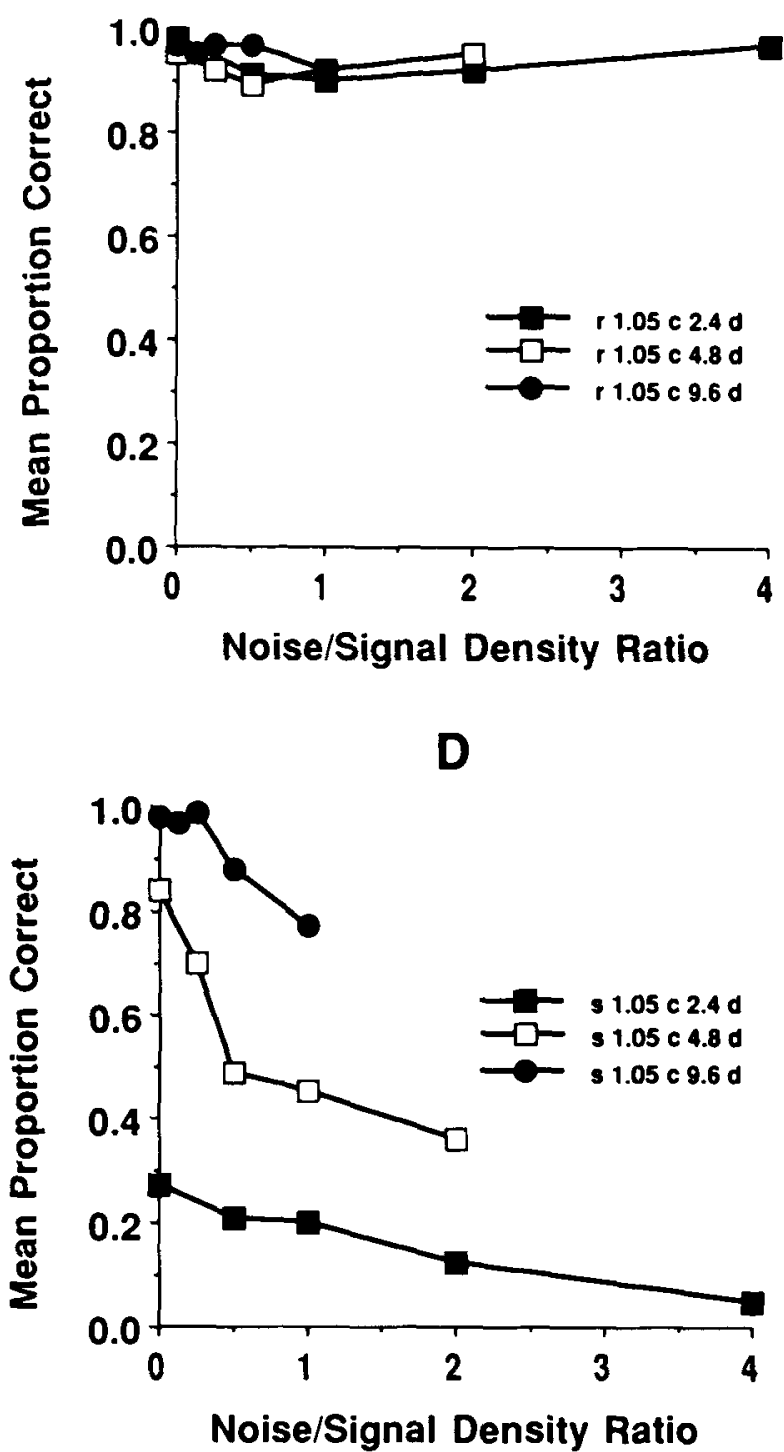

Figure 7. Mean proportion correct as a function of noise/signal density ratio for random (A and $B$ ) and surface (C and D) conditions. Separate curves are plotted for different random ( $r$ ), surface (s), corrugations (cycles per degree; c), and surface density (dots per degree'; d).

expect greater detection accuracy for noise points that form surfaces than for noise points randomly positioned in a volume.

\section{Method}

Subjects. The subjects were 4 graduate students at the University of California, Riverside who were paid for their participation. Two of the subjects had participated in Experiments 1,2,3, and 4. Three of the subjects were naive with regard to the purpose of the experiment.

Design. Five independent variables were examined: type of display (corrugated surface or random volume), noise type (overlapping or nonoverlapping), noise configuration (random volume or frontoparallel surface), the density of the noise points $(0,2.4,4.8$,
9.6, and $\left.19.2 \mathrm{dots} / \mathrm{deg}^{2}\right)$, and the spatial frequency of the corrugated surface $(0.52$ or $1.05 \mathrm{cpd})$.

Stimuli. The stimuli were similar to the displays used in the previous experiments. The displays simulated a 3-D corrugated surface or a random 3-D volume specified by optic flow. The surface and random velocities were generated in the same manner as in the prior experiments. The density of the surface and random displays was $9.6 \mathrm{dots} / \mathrm{deg}^{2}$. The maximum and minimum velocities for the surface and random conditions were $2.84 \% \mathrm{sec}$ and $1.95 \% \mathrm{sec}$ (identical to all prior experiments).

Combinations of the levels of noise type and noise configuration resulted in four conditions: overlapping volume noise, overlapping surface noise, nonoverlapping volume noise, and nonoverlapping surface noise. For the overlapping surface noise condition, the velocities were $1.95 \% \mathrm{sec}$ and $2.84 \% \mathrm{sec}$. For the overlapping volume 


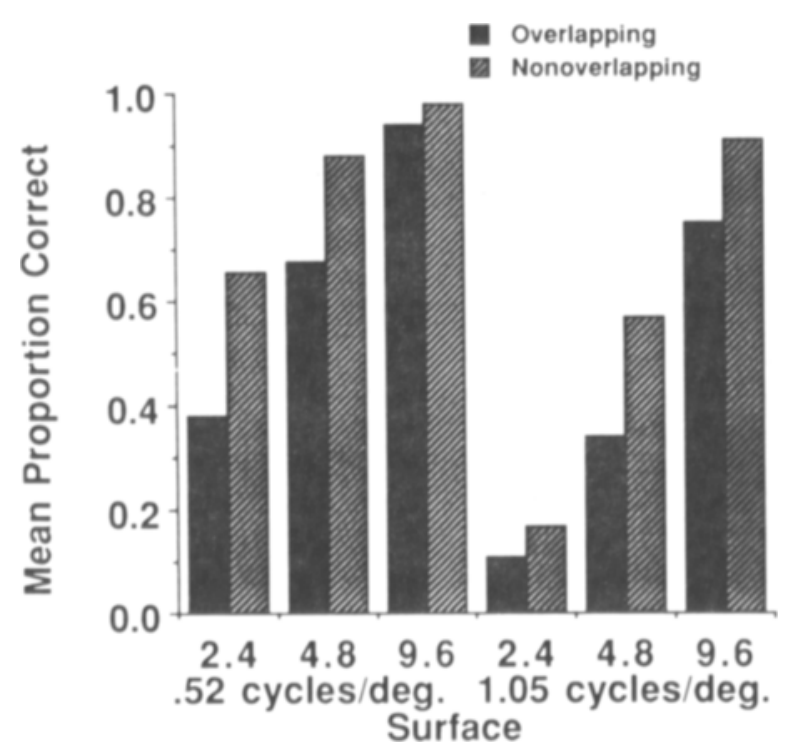

Figure 8. Bar graph of the mean proportion correct as a function of different combinations of surface density $(2.4,4.8$, and $\left.9.6 \mathrm{dots} / \mathrm{deg}^{2}\right)$ and corrugation frequency $(.52$ and $1.05 \mathrm{cpd})$. Solid bars show results for overlapping noise (Experiment 2) and striped bars shows results for nonoverlapping noise (Experiment 4).

noise condition, two random-volume fields were generated, with each field centered about the value of the two surface noise velocities. The velocities for the two random volumes were $2.675 \% \mathrm{sec}$ and $2.925 \% \mathrm{sec}$, and $1.865 \% \mathrm{sec}$ and $2.115 \% \mathrm{sec}$, respectively. For the nonoverlapping surface noise condition, the velocities were $0.76 \% \mathrm{sec}$ and $4.04 \% \mathrm{sec}$. For the nonoverlapping volume noise, the velocities were $0.635 \% \mathrm{sec}$ and $0.885 \% / \mathrm{sec}$, and $3.915 \% \mathrm{sec}$ and $4.165 \% / \mathrm{sec}$, respectively.

Procedure. The procedure that was used in Experiment 1 was used, with the following exception: each subject was run through 25 blocks of trials over a 3-day period.

\section{Results and Discussion}

The results are presented in Figure 11. Each data point is the average of 25 responses from 5 subjects. As is shown in Figures 11A and 11B, the subjects were consistently correct in detecting the random displays. These results are virtually identical to the results obtained in Experiments 1-4.

For surface display trials (shown in Figures $11 \mathrm{C}$ and 11D), the mean proportion correct decreased with an increase in the noise/signal density ratio of the display. The findings for the noise/signal density ratio and spatial frequency of the corrugated surface are remarkably similar to the results obtained in the first four experiments. The mean proportions correct for the $0,0.25,1$, and 2 noise/signal density ratios were $.99, .97, .83$, and .61 , respectively. In addition, greater detection performance occurred for the low (Figure 11C) than for the high (Figure 11D) spatial frequency of the corrugated surface. The mean proportions correct for the 0.52 - and 1.05 -cpd corrugations were .93 and .78 , respectively.

There was little difference in detection performance for the two types of noise configuration. The mean propor- tions correct for the surface and volume noise conditions were .85 and .86 , respectively. However, greater detection performance occurred for the nonoverlapping than for the overlapping noise. The mean proportions correct for the nonoverlapping and overlapping noise conditions were .91 and .79 , respectively. The effect of noise type varied as a function of the noise/signal density ratio. As depicted in Figures 11C and 11D, the difference in performance between nonoverlapping and overlapping noise increased with an increase in the noise/signal density ratio. This difference was most pronounced at the 2.0 noise/ signal density level, with mean proportions correct (averaged across corrugation frequency and noise configuration) of .64 and .23 for nonoverlapping and overlapping noise, respectively. These results provide support for the conclusion-based on the comparison of results from Experiments 2 and 4 -that detection accuracy is greater for nonoverlapping than for overlapping noise.

\section{GENERAL DISCUSSION}

The present results demonstrate that subjects can detect the presence of a surface from optic flow with greater accuracy when the distribution of surface velocities does not overlap with the distribution of noise velocities. This result occurred across variations in the noise/signal density ratio and in the frequency of the corrugated surface. These results support the hypothesis that the detection of a surface in the presence of noise may be accomplished by an analysis based on the distributions of velocity present in the display. According to this approach, when the distribution of noise velocities does not overlap the distribution of surface velocities, the observer can more easily segregate the velocities due to the separation of velocities of surface and noise points.

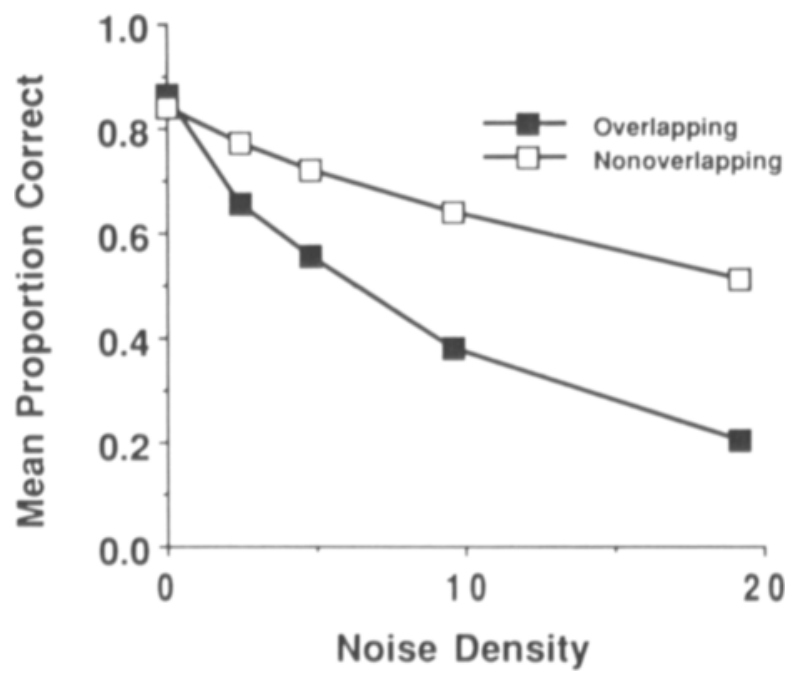

Figure 9. Mean proportion correct as a function of noise density (dots per degree ${ }^{2}$ ). Plotted are two curves for the results for overlapping and nonoverlapping noise examined in Experiments 2 and 4, respectively. 

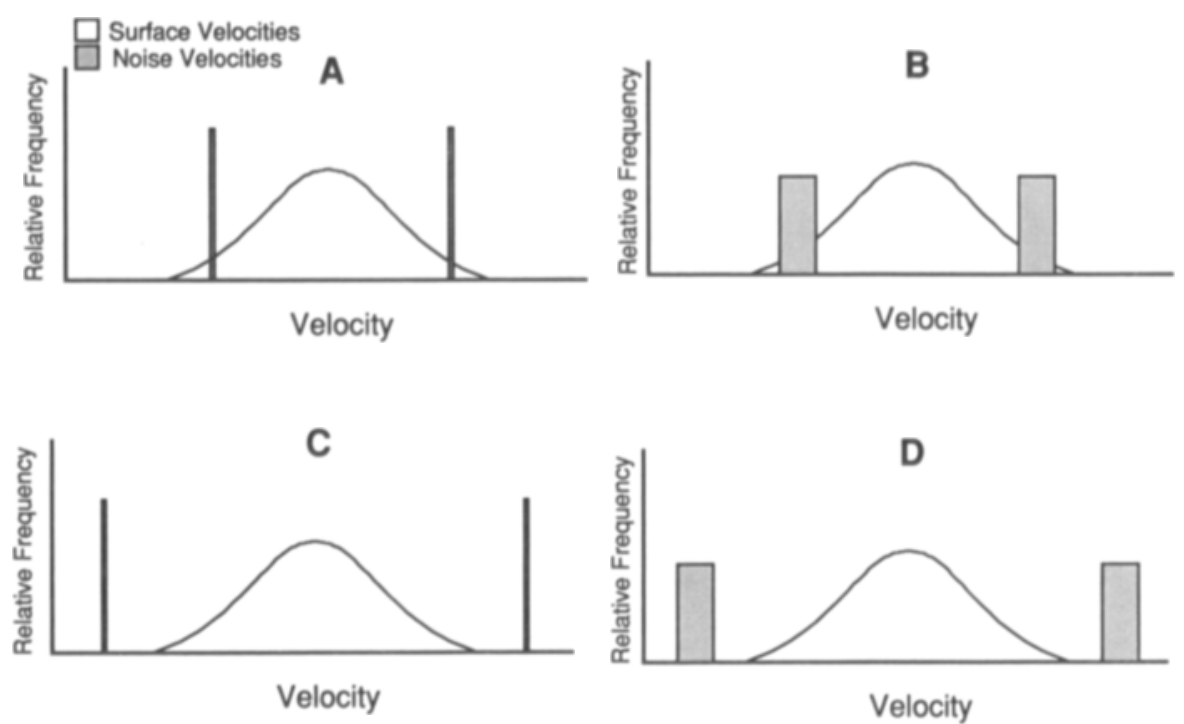

Figure 10. The four types of display conditions examined in Experiment 4 , illustrated in terms of theoretical distributions of velocities present in the display. Panels A, B, C, and D are distributions for display conditions of overlapping surface noise, overlapping volume noise, nonoverlapping surface noise, and nonoverlapping volume noise, respectively.

The present results also suggest that an analysis based on velocity distributions is useful within a limited range of noise/signal density ratios. The subjects had difficulty detecting the presence of a surface when the number of noise points greatly exceeded the number of surface points. For example, detection performance, across variations in noise type and corrugation frequency, was below chance when the noise/signal density ratio was 4 .

Previous research (Andersen, 1989) suggests that another limitation to this type of analysis is the number of distributions that can be segregated. Subjects were presented with motion parallax displays simulating overlapping transparent frontoparallel surfaces that translated horizontally or along the depth axis. As discussed earlier, they were able to reliably detect up to three different surfaces, regardless of the type of translation. If an analysis based on distributions of velocities was employed, then the results suggest that only three distributions, specifying each surface, can be segregated at any given time. An important goal for future research is to examine this limit for more complex surfaces (e.g., curved surfaces).

The present results also demonstrate that surface detection in the presence of noise was not solely dependent on the noise/signal density ratio. Variations in the corrugation frequency resulted in different patterns of performance across different noise/signal density ratios. This occurred across variations in surface density and noise type in all four experiments. These findings provide support for the conclusion of other research (Andersen, 1992) - that change in surface curvature is important for the detection of 3-D surfaces.
Another factor that is important for surface detection in the presence of noise is the density of the surface. Different levels of detection accuracy were found in Experiments 2 and 4 for equivalent noise/signal density ratios when different surface densities were employed. Specifically, greater accuracy occurred for higher than for lower surfaces densities across equivalent noise/signal density ratios. This pattern of results occurred for both overlapping and nonoverlapping noise. It is important to note that this finding is not due to different levels of discrimination accuracy resulting from different levels of surface density. Examination of the results for the .53-cpd surfaces (see Figures 5 and 7) indicate that, when noise points were absent (noise/signal density ratio of 0 ), the subjects had near-perfect accuracy across all three surface densities examined. As the noise/signal density ratio increased, detection performance declined at a greater rate for lower surface density levels than for higher surface density levels. This difference in performance was greatest for the nonoverlapping noise (see Figure 7). An important issue for future research will be to determine why different levels of performance occur for different levels of surface density across different levels of noise/signal density.

Finally, the present results indicate a remarkable tolerance to noise in the detection of 3-D surfaces. The subjects demonstrated above-chance performance for conditions in which the noise/signal density ratio was 2 (see Figures 4 and 6). The present results indicate that subjects are capable of detecting the presence of a surface when smoothness of the velocity field is severely violated. 

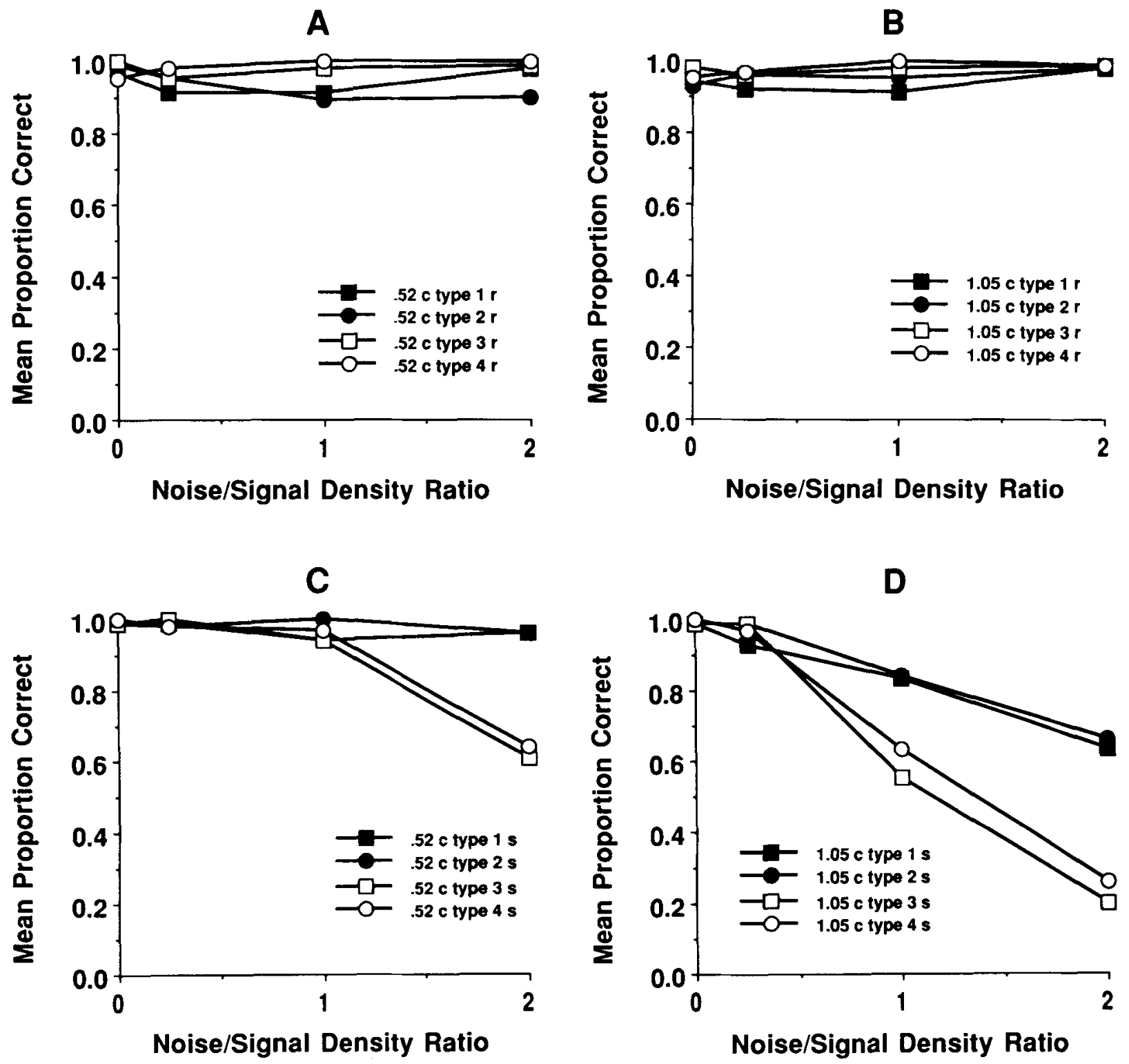

Figure 11. Mean proportion correct as a function of noise/signal density ratio for random ( $A$ and $B$ ) and surface (C and D) conditions. Figures $C$ and $D$ show results for the .53- and 1.05-cpd corrugation conditions. Type 1, 2, 3, and 4 in the legends refer to nonoverlapping surface noise, nonoverlapping volume noise, overlapping surface noise, and overlapping volume noise, respectively.

In summary, the present results demonstrate that human observers can detect the presence of a surface when considerable noise is present in the display and suggests that this may be accomplished by segregating noise points and surface points based on the distributions of velocities present in the display. In addition, the density and corrugation frequency of the surface are important factors in surface detection in the presence of noise.

\section{REFERENCES}

Adelson, E. H., Movshon, J. A. (1982). Phenomenal coherence of moving visual patterns. Nature, 300, 523-525.

AnDersen, G. J. (1989). Perception of three-dimensional structure from optic flow without locally smooth velocity. Joumal of Experimental Psychology: Human Perception \& Performance, 15, 49-55.

ANDERSEN, G. J. (1991). Interpolation of smooth 3-D shape from motion. Investigative Ophthalmology \& Visual Science, 32(Suppl. 3), 1277.

ANDERsen, G. J. (1993). Detection of 3-D surfaces from optic flow. Manuscript submitted for publication.

Braunstein, M. L., \& ANDersen, G. J. (1981). Velocity gradients and relative depth perception. Perception \& Psychophysics, 29, 145-155.

Braunstein, M. L., Payne, J. W. (1969). Perspective and form ratio as determinants of relative slant judgments. Journal of Experimental Psychology, 81, 584-590.

Cornilleau-Pérès, V., \& Droulez, J. (1989). Visual perception of surface curvature: Psychophysics of curvature detection induced by motion parallax. Perception \& Psychophysics, 46, 351-364. 
De BruYn, B. (1992). Segregation of spatially superimposed optic flow components. Manuscript submitted for publication.

EBY, D. W. (1992). The spatial and temporal characteristics of perceiving 3-D structure from motion. Perception \& Psychophysics, 51 , 163-178.

Gerbino, W., \& Bernetti, L. (1984). One, two, many: Textural segregation on the basis of motion. Perception, 13, 38.

GiBson, J. J. (1950). The perception of the visual world. Boston: Houghton Mifflin.

GiBson, J. J. (1966). The senses considered as perceptual systems. Boston: Houghton Mifflin.

Grimson, W. E. L. (1981). From images to surfaces: A computational study of the human early visual system. Cambridge, MA: MIT Press.

HeLmholtz, H. L. F. voN (1962). Treatise on physiological optics (Vol. 3, J. P. C. Southall, Ed. and Trans.). New York: Dover. (Original work published 1867)

KoenderinK, J. J. (1986). Optic flow. Vision Research, 26, 161-180.

Koenderink, J. J., \& VAN Doorn, A. J. (1976). Local structure of movement parallax of the plane. Journal of the Optical Society of America, 66, 717-723.

Longuet-Higgins, H. C., \& Prazdny, K. (1980). The interpretation of a moving retinal image. Proceedings of the Royal Society of London: Series B, 208, 385-397.

Norman, J. F., \& LaPPIN, J. S. (1992). The detection of surface curvatures defined by optical motion. Perception \& Psychophysics, 51, 386-396.

Rogers, B., \& Graham, M. (1979). Motion parallax as an independent cue for depth perception. Perception, 8, 125-134.

SNOWDEN, R. J. (1990). Suppressive interactions between moving patterns: Role of velocity. Perception \& Psychophysics, 47, 74-78.

ToDD, J. T. (1984). The perception of three-dimensional structure from rigid and nonrigid motion. Perception \& Psychophysics, 36, 97-103.
Todd, J. T. (1985). Perception of structure from motion: Is projective correspondence of moving elements a necessary condition? Journal of Experimental Psychology: Human Perception \& Performance, 11 , 689-710.

UTrAL, W. R. (1975). An ausocorrelation theory of form detection. Hillsdale, NJ: Erlbaum.

UTTAL, W. R. (1983). Visual form detection in 3-dimensional space. Hillsdale, NJ: Erlbaum.

UTTAL, W. R. (1985). The detection of nonplanar surfaces in visual space. Hillsdale, NJ: Erlbaum.

UTTAL, W. R. (1987). The perception of dotted forms. Hillsdale, NJ: Erlbaum.

UtTAL, W. R. (1988). On seeing forms. Hillsdale, NJ: Erlbaum.

UtTal, W. R., Davis, N. S., Welke, C., \& Kakarala, R. (1988) The reconstruction of static visual forms from sparse dotted samples Perception \& Psychophysics, 43, 223-240.

VAN Doorn, A. J., Koenderink, J. J. (1982a). Spatial properties of the visual detectability of moving spatial white noise. Experimental Brain Research, 45, 189-195.

van DoORn, A. J., \& Koenderink, J. J. (1982b). Temporal properties of the visual detectability of moving spatial white noise. Experimental Brain Research, 45, 179-188.

voN KRIES, J. (1962). Notes on the perception of depth. In H. L. F. von Helmholtz, Treatise on physiological optics (J. P. C. Southall, Ed. and Trans., Vol. 3). New York: Dover. (Original work published 1910)

(Manuscript received July 27, 1992; revision accepted for publication February 1, 1993.) 